\title{
Relação entre os condicionamentos operante e respondente no transtorno obsessivo-compulsivo
}

\author{
Operant and respondent conditionings relation \\ in obsessive-compulsive disorder
}

\author{
Paulo Roberto ABREU \\ Cynthia Granja PRADA²
}

\begin{abstract}
Resumo
As duas primeiras versões do Diagnostic and Statistical Manual Disorders, nos anos de 1952 e 1968, continham definições inespecíficas e sucintas sobre o transtorno obsessivo-compulsivo. Somente a partir da terceira edição, em 1980, é que foram definidos critérios diagnósticos mais acurados sobre esse transtorno e os seus sintomas. Embora a ciência tenha dado algum avanço nos estudos, a literatura ainda apresenta grande dificuldade em clarificar o transtorno obsessivo-compulsivo sob todas as suas nuanças. Semelhante impasse vive ainda a análise do comportamento, a despeito de ter sido a teoria que mais teve a oferecer ao fenômeno. O objetivo deste ensaio é analisar sob os pressupostos do behaviorismo radical o caso de uma cliente atendida durante estágio clínico. A partir da análise de caso foi lançada uma hipótese sobre os eventos distais implicados no condicionamento dos comportamentos relacionados ao transtorno. Concluiu-se que a instalação dos comportamentos obsessivo-compulsivos da cliente se justifica frente aos condicionamentos operante e respondente por que passou.
\end{abstract}

Palavras-chave: análise do comportamento; ansiedade; psiquiatria; transtorno obsessivo-compulsivo.

\begin{abstract}
The first two editions of Diagnostic and Statistical Manual Disorders in 1952 and 1968 presented unspecific and brief definitions about the Obsessive-Compulsive Disorder. Only after the third edition in 1980 more accurate diagnostic criteria about its symptoms were defined. Although the knowledge about the disorders has improved by the studies, the scientific literature has had difficulties to follow up all its particularities. The behavior analysis has been the approach which has contributed most to this disorder treatment and comprehension, although it has also suffered the same problems. The goal established in this paper was to analyze a case of a client by the radical behaviorism approach. From this case analysis, a hypothesis was raised about the distal events involved in the behavior conditioning related this disorder. It was possible to conclude that the installation of the client's obsessive-compulsive behavior can be justified by operant and respondent conditionings.
\end{abstract}

Key words: behavior analysis; anxiety; psychiatry; obsessive-compulsive disorder.

O transtorno obsessivo-compulsivo (TOC) é considerado o quarto transtorno psiquiátrico mais freqüente, tendo uma taxa de prevalência na vida de aproximadamente $2 \%$ em várias partes do mundo

$\mathbf{\nabla} \mathbf{\nabla v}$

1 Curso de Psicologia, Universidade Federal do Paraná. Praça Santo André, 50, $1^{\circ}$ Andar, Edifício Central, 80020-300, Centro, Curitiba, PR, Brasil. Correspondência para/Correspondence to: P.R. ABREU. E-mail: <pauloabreu_ufpr@yahoo.com.br>.

2 Doutoranda em Educação Especial, Universidade Federal de São Carlos. São Carlos, SP, Brasil.

Agradecimentos: aos psicólogos Bruno Ângelo Strapasson e Renata Rolim Sakiyama pela revisão do texto. 
(Torres \& Prince 2004). Seus índices epidemiológicos o deixam atrás apenas das fobias, transtornos relacionados a substâncias e transtorno depressivo maior (Del-Porto, 2001). A despeito de ainda não ter sido encontrado um entendimento sólido para a etiologia do TOC (Miguel, Rauch \& Jenike, 1998; Gonzalez, 1999; Parmet, Glass \& Glass, 2004; Ronchetti, Böhme \& Ferrão, 2004), a psiquiatria já teceu suas hipóteses, principalmente a partir dos estudos de neuroimagem (Valente \& Filho, 2001; Lacerda, Dalgalarrondo \& Camargo, 2001; Pujol, Soriano-Mas, Alonso, Cardorner, Menchón, Deus \& Vallejo, 2004), de neuroquímica (Marazziti \& Di Nasso, 2000; Marques, 2001) e da genética relacionada ao transtorno (Gonzalez, 1999; 2001).

Os estudos de neuroimagem, ainda que se constituam de métodos de investigação tidos em alto conceito dentro das neurociências, pouco contribuíram para a compreensão da patogênese do TOC. As pesquisas de neuroimagem estrutural (imagem por ressonância magnética), que poderiam apontar possíveis alterações anatômicas nas áreas cerebrais do circuito fronto-estriado-tálamo-cortical relacionado à fisiopatologia doTOC (Ronchetti et al., 2004), mostraram resultados inconclusivos até o presente momento (Lacerda et al., 2001; Valente \& Filho, 2001). Devido à heterogeneidade das alterações mapeadas nos diferentes estudos não foi possível achar um padrão patológico comum. Os estudos de neuroimagem funcional, representados em sua maior parte pela positron emission tomography (PET) e pela single photon computed emission tomography (SPECT), apresentaram resultados mais promissores do que os achados estruturais. Os dados convergem para alterações no nível de glicose no córtex orbitofrontal (Lacerda et al., 2001). Alterações sugeridas em estudos de outros locus do circuito fronto-estriado-tálamo-cortical, a exemplo dos gânglios da base, não forneceram dados universais. Há relatos de atividade normal nessa região cerebral em algumas amostras de pacientes (Bussato, Zamignani, Buchpiguel, Garrido, Glabus \& Rocha, 2000).

A segunda hipótese, pertinente às alterações dos níveis de certos neurotransmissores, é sustentada pelos achados relativos ao sistema serotoninérgico. Tal hipótese deriva de estudos acerca do tratamento farmacológico do TOC, mostrando forte correlação 226 positiva entre a melhora dos sintomas obsessivo- -compulsivos em pacientes tratados com os inibidores de recaptação de serotonina (IRS), como a sertralina, paroxetina, citalopram ou fluvoxamina nos IRS seletivos, e como a clomipramina, nos IRS não seletivos (Marazziti \& Di Nasso, 2000). Os fármacos que agem sobre a noradrenalina mostraram-se pouco eficazes, o que parece colocar o TOC em um patamar diferenciado dos outros transtornos nos quais o sistema serotoninérgico esteja preferencialmente envolvido, a exemplo da depressão e do transtorno do pânico (Marques, 2001).

A terceira hipótese psiquiátrica abordada está fortemente atrelada às duas primeiras, visto que variações genéticas podem ter como conseqüência mudanças metabólicas e neuroquímicas, características do transtorno. Conquanto os estudos moleculares venham apresentando avanços promissores na busca dos genes envolvidos na etiologia do TOC (Gonzalez, 1999; 2001), os métodos de investigação que realmente alcançaram resultados significativos foram os estudos de família e de gêmeos. Os estudos de família mostraram maior prevalência deTOC, sintomas obsessivo-compulsivos e outros transtornos normalmente comórbidos, como a síndrome de Tourett e a síndrome de tique motor ou vocal entre parentes dos indivíduos afetados (Gonzalez, 1999; 2001). Já os estudos de gêmeos demonstraram uma concordância de $80 \%$ a $87 \%$ entre gêmeos monozigóticos e de 47\% a 50\% em gêmeos dizigóticos (Jenike, 2004). Outros aspectos que indicariam uma forte correlação genética seriam as taxas semelhantes de prevalência nas diferentes culturas, a idade de início e as comorbidades normalmente diagnosticadas em pacientes com o TOC (Del-Porto, 2001).

Procurar a etiologia do TOC na plataforma biológica do organismo como tradicionalmente vem fazendo a psiquiatria parece ter sido um método insuficiente na explicação do fenômeno. A psiquiatria estabelece critérios sintomatológicos, pautados na topografia das respostas dos pacientes com o transtorno (Zamignani, 2001a; Gongora, 2003). Esse modelo é denominado quase-médico posto que, ao contrário das outras patologias médicas que não as psiquiátricas, faltam especificações sobre a etiologia da doença na qual se possa afirmar que seus sintomas sejam uma manifestação direta (Cavalcante \&Tourinho, 1998). 
O modelo analítico-comportamental sustenta que se observe nas variáveis ambientais externas as condições necessárias para a instalação e manutenção do comportamento (Tourinho, 1999; Tourinho, Texeira \& Maciel, 2000). Diferentemente da psiquiatria que aborda os problemas de comportamento em termos de sintomas decorrentes de uma disfunção anátomo-fisiológica, a análise do comportamento os concebe como sendo déficits e/ou excedentes comportamentais relativos ao contexto social em que ocorrem (Del-Prette \& BolsoniSilva, 2003), produtos dos três níveis de variação e seleção de comportamentos, a saber, da história filogenética da espécie homo sapiens, da história ontogenética do indivíduo e da cultura em que esse se encontra (Skinner, 1981/1984; 1990). No nível ontogenético, do qual a análise do comportamento preferencialmente se ocupa, é importante que se observem os eventos antecedentes ao comportamento-problema de análise e as conseqüências que se seguiram à sua emissão (Sério, Andery, Gioia \& Micheletto, 2004). Conveniou-se chamar essa relação entre eventos de tríplice contingência (Skinner, 1953/1965), na qual um dado comportamento de análise é entendido como sendo função dos eventos antecedentes e conseqüentes do contexto em que ocorre. Portanto, mais importante do que o mapeamento das topografias dos comportamentos-problema relativos ao "transtorno"é o mapeamento das variáveis das quais esses são função (Cavalcante \& Tourinho, 1998).

As alterações fisiológicas propostas pela psiquiatria parecem não ser a causa, mas a conseqüência das variáveis ambientais que já vêm atuando na história do organismo (Marinho, 2001). Segundo Skinner (1990), a fisiologia seria o produto dos três níveis de variação e seleção, sendo, portanto, secundária às contingências de sobrevivência filogenéticas, às contingências de reforçamento ontogenéticas e às contingências especiais mantidas pelo ambiente social. De posse desse entendimento, o behaviorismo radical não concede status causal aos eventos privados, do qual a fisiologia faz parte. As respostas fisiológicas são comportamentos e como tais não são a explicação para a causa do fenômeno comportamental, mas parte daquilo que precisa ser explicado (Skinner 1953/1965; 1990). As variações serotoninérgicas e as variações metabólicas do córtex órbito-frontal encontradas nos estudos neuroquímicos e de neuroimagem funcional respectivamente não acrescentam quase nada na elucidação da etiologia do TOC. Dadas modificações parecem ser o efeito das contingências de reforçamento em curso. Evidências corroborando essa hipótese aparecem da constatação da normalização do metabolismo cerebral em uma ou mais áreas que compõem o circuito pré-frontal-estriado-tálamo-cortical em pacientes tratados com a técnica comportamental de exposição com prevenção de respostas (Lacerda, 2000).

No diálogo entre as duas ciências, cabe ainda uma relativização da utilidade e validade da hipótese psiquiátrica relacionada à genética do transtorno. Skinner (1953/1965) chamou a atenção para a importância da genética do comportamento no estudo da extensão em que as disposições comportamentais são herdadas. O autor considera que para o entendimento da variação e seleção do repertório comportamental de um organismo é importante que se atente também para o nível filogenético de análise (Skinner, 1984; 1990). O conhecimento das limitações biológicas do organismo tornaria possível uma melhor utilização dos métodos de predição e controle analítico-comportamentais (Skinner, 1953/1965; 1976). Conquanto reconhecesse essa importância, reservou à filogênese a evolução da suscetibilidade aos estímulos reforçadores incondicionados no condicionamento operante (Skinner, 1989/2002). Deixou patente a possibilidade de se atribuir o comportamento operante apenas à carga genética do organismo (Skinner 1953/1965; 1989/2002), visto que a expressão fenotípica não se faz sem a participação das contingências de reforçamento ontogenéticas.

Os estudos em genética são ainda muito pobres na determinação da influência do ambiente no TOC. Del-Porto (2001) apontou para a influência do meio na "moldagem" de certos sintomas, a exemplo das obsessões de agressão na forma de blasfêmias e sacrilégios nas culturas religiosas e a exemplo de uma maior incidência das obsessões de cunho sexual nos países que impedem a expressão da sexualidade. Os índices estatísticos encontrados podem não exprimir o efeito de uma causa orgânica comum, mas o efeito de ambientes semelhantes na modelagem de comportamentos com igual topografia e funcionalidade. Sobre o tópico, Skinner (1974/1976) pontuou que a universalidade genético-comportamental mereceria ser ponderada, pois o que também se mostra bastante 
semelhante no fenômeno são as contingências similares a que cada espécie está submetida. A observação da modelagem de comportamentos obsessivo-compulsivos sob contingências de controle aversivo encontradas em culturas coercitivas se mostra de grande utilidade no entendimento do fenômeno (Abreu \&Prada, 2004)

O presente estudo de caso lança luz à instalação dos comportamentos obsessivo-compulsivos ao analisar o histórico de condicionamentos operante e respondente de uma cliente com TOC. Para tanto, dar-se-á ênfase aos eventos distais ocorridos em sua vida, não contemplando, portanto, o relato e a análise pormenorizados das intervenções realizadas.

\section{Estudo de Caso}

O estudo fez-se através dos atendimentos clínicos realizados no período de 27/1/2003 a 12/12/2003. As sessões aconteceram semanalmente nas instalações da clínica-escola da Universidade Federal do Paraná, sendo acompanhadas através do espelho unidirecional por outro integrante da equipe. A cliente atendida, que será chamada de "O", é uma senhora com 55 anos, casada e mãe de três filhas (22, 31 e 33 anos de idade), todas casadas. "O" é dona de casa mas também vende bombons. Na ocasião do início do tratamento, sua filha mais nova ainda residia com a cliente por ser a única filha solteira. Posteriormente casou-se, mudando para outra residência.

\section{Queixas}

Problemas com o cônjuge: o cônjuge - que será chamado de "A"- estava aposentado havia oito meses e por isso permanecia a maior parte do tempo em casa. "A" foi descrito pela esposa como sendo uma pessoa que não tinha iniciativa alguma. "O" contou que o parceiro não arrumava as coisas dentro de casa, deixando os cômodos todos desorganizados. Quando tentava fazer algum serviço doméstico, passava horas trabalhando, finalizando em um serviço incompleto. Mais que isso, sujava muito a casa tentando fazê-lo. A cliente trouxe também que normalmente evitava ficar na presença do marido.

Problemas com os genros: relatou seu descontentamento em relação aos genros. O marido de sua filha mais velha foi descrito como alguém que não tinha raízes, uma pessoa que não possuía uma adequada ligação afetiva com a sua família de origem. Segundo a cliente, o genro não conseguia emprego estável e ainda omitiu de sua esposa o fato de que já tinha tido um filho com outra mulher. Por seu turno, a filha do meio se casou com alguém com problemas semelhantes - além de não ter raízes e emprego estável, esse genro não cuidava adequadamente da sua esposa enferma. Dessas constatações, "O"tentou direcionar as decisões das filhas em relação aos maridos, chegando a empregar grande esforço para convencer uma das filhas a pedir a separação.

Problemas com o estado de saúde da filha enferma: "O" apresentou preocupação crescente com uma das filhas. A família prontamente desaprovou a intensidade de seus incômodos. Embora tenha uma doença crônica (deficiência de imunoglobina), a filha vinha honrando todos os seus deveres. Trabalhava de dia em período integral e cursava faculdade à noite. "O" afirmou que a doença logo iria consumir a vitalidade da filha, pontuando que essa era também a percepção do médico especialista. Posteriormente afirmou que o médico nada Ihe disse a respeito do prognóstico da doença. O profissional apenas se limitou a afirmar que o problema era sério.

Ataques de pânico: a cliente relatou ter tido ataques de pânico recorrentes. Os ataques ocorriam no momento em que despertava do sono e, embora estivessem circunscritos a esse contexto, não aconteciam invariavelmente em todas as situações.

Problemas com o trabalho: "O" trouxe que estava muito cansada e ansiosa devido ao trabalho excessivo. No $20^{\circ}$ atendimento expressou que desejava mudar seu cotidiano, mas que não conseguia porque se sentia mais calma quando trabalhava. Nessa sessão colocou ainda que sempre que terminava um serviço, já começava a pensar em possíveis outros trabalhos para fazer, como rebocar uma parede, arrumar um portão, varrer a casa etc.

Obsessões: mais tarde, no $24^{\circ}$ atendimento, relatou que no auge de sua ansiedade era acometida por pensamentos que Ihe diziam que algo ruim estaria para acontecer com sua família e/ou que teria um derrame ou um ataque do coração. Segundo a cliente, os pensamentos pareciam avisos. Ocorriam-Ihe ao menos cinco vezes por dia. 


\section{Algumas intervenções de interesse para a avaliação funcional}

No quinto atendimento foi proposto à cliente que preenchesse uma lista com as suas atividades semanais. Durante a análise conjunta, concluiu-se que a cliente reservava um limitado tempo para si. Relatou que por mais que gostasse de uma novela, somente a assistia duas vezes por semana e logo já saía porque tinha que costurar para as filhas. Todo o restante da semana era destinado aos serviços domésticos e à venda de bombons, guardando somente quatro horas semanais para estar com o marido." $\mathrm{O}$ "trabalhava desde o momento em que acordava até a hora de dormir.

O curso dos ataques de pânico teve seu crescimento gradativamente interrompido com o progresso da terapia. Apenas no quarto atendimento relatou ter tido outro ataque, logo após uma piora no estado de saúde da filha.

\section{Eventos distais}

Na sétima sessão foi proposta à cliente uma vivência com o objetivo de elucidar os eventos distais responsáveis pelo aumento de sua ansiedade. Nela, "O" descreveria o que viveu em algumas épocas de sua vida.

Com nove anos a cliente foi morar com a irmã mais velha, posto que sua família estava passando por sérias dificuldades financeiras. Inicialmente sua irmã lhe tratava bem, sendo companheira e solícita nos momentos de dificuldade. Com o nascimento de seus filhos o relacionamento mudou. "O" começou a sofrer violência física e verbal. Era incumbida pela irmã dos serviços da casa e da guarda dos sobrinhos. A irmã lhe violentava ao constatar que os serviços haviam sido feitos de forma precária ou ainda quando constatava que "O"não havia cumprido as tarefas impostas. Quando a punia fisicamente, puxando seus cabelos, também Ihe agredia verbalmente. Nas agressões, chamava-a de "incompetente", exprimindo que "O" não tinha capacidade para corresponder às tarefas que lhe eram incumbidas. Assim, a cliente tentava realizar as tarefas domésticas melhor do que fizera, objetivando esquivar-se das punições. Embora o fizesse, ainda assim era punida. Novamente se esforçava para desempenhar melhor os serviços, ainda conforme as reclamações da irmã, mas outra vez era punida. O encadeamento tornou-se cíclico. A cliente concluiu em atendimento que era punida independentemente da apresentação de um trabalho conforme as especificações da irmã.

\section{Discussão}

Ao longo das entrevistas e intervenções foi verificado que a cliente estava tendo respondentes ansiosos intensos e que para esquivar das situações eliciadoras, punha-se a trabalhar compulsivamente desde a hora em que acordava até a hora em que se retirava para dormir. A ansiedade era eliciada pelos fatores de estresse que estava vivenciando em sua vida, como os problemas conjugais, as preocupações com as filhas, os problemas com os genros, o medo da incidência de mais um ataque de pânico, o montante de trabalho e as obsessões. Esses comportamentos preenchiam os critérios sintomatológicos do DSM-IV-TR (American Psychiatric Association, 2003) para o TOC com ataques de pânico associados. Tratava-se de obsessões e compulsões diferentes dos padrões sintomáticos categorizados na literatura psiquiátrica a partir da Yale-Brown Obsessive Compulsive Scale (Y-BOCS) (Hounie, Brotto, Diniz, Chacon, \& Miguel, 2001).

O condicionamento da ansiedade em "O"seguiu o paradigma proposto por Estes e Skinner (1941) e revisitado por Lundin (1977). Inicialmente o estímulo neutro "casa desorganizada/suja" foi pareado com o estímulo aversivo primário "violência física". A seguir, o estímulo neutro se tornou um estímulo aversivo secundário. Após o condicionamento, a próxima operação de apresentação dos estímulos ocorreria da seguinte forma: primeiramente o estímulo aversivo secundário que indica a vinda contígua do aversivo primário "violência física" seria novamente apresentado. Como não existia a possibilidade de fuga ou esquiva desse aversivo, pois " $\mathrm{O}$ " inevitavelmente seria punida, ocorreu o eliciamento da ansiedade no tempo transcorrido entre a apresentação do aversivo secundário e a apresentação do aversivo primário.

Nessa época, a função dos comportamentos de organização/limpeza emitidos por "O" era se esquivar das punições da irmã. Não havia outras alternativas cabíveis. A mãe não concordava com o seu retorno para a casa e se não fizesse os serviços prescritos ou os apresentasse mal feitos por conta de uma revolta, 
provavelmente teria como conseqüência uma punição muito mais intensa. A única forma de conseguir se esquivar com sucesso seria emitir os comportamentos estabelecidos pela irmã.

Reese (1978, p.85) colocou que:

Um estímulo aversivo forte, como o ruído de uma explosão próxima, suprirá o comportamento operante que estiver sendo emitido. Eliciará também um susto respondente, aumento de pulsação, aceleração cardíaca e outros reflexos de glândulas e músculos lisos. Em casos extremos, o organismo pode urinar e defecar. Estes respondentes são incompatíveis com o comportamento operante, e, assim, interferem no mesmo.

No caso de "O", os comportamentos de esquiva constituíam o próprio operante discriminado em curso que vinha sendo punido pela sua irmã mais velha. Ao contrário do que normalmente ocorre com os outros operantes em curso, esse operante não foi suprimido pelos respondentes emocionais incompatíveis. Quais variáveis então estiveram mantendo a emissão desses comportamentos?

Uma análise demonstrou que o operante discriminado resistente à extinção foi provavelmente reforçado sob esquema intermitente. Acrescido a isso, existiam outras variáveis que estariam influenciando a freqüência e a intensidade com que "O" emitia as respostas compulsivas: a observada modificação gradual na intensidade e na freqüência de seus comportamentos de esquiva foi também conseqüência das operações aversivas em curso, ou seja, do histórico das punições contingentes à constatação de uma não adequação dos serviços domésticos. Essas operações, também chamadas de operações estabelecedoras, aumentaram ainda mais o efeito do reforçamento negativo sob esquema intermitente (Catania, 1999). Com o reforçamento, essas respostas tiveram suas freqüências gradativamente aumentadas. Hipotetiza-se que os Doforços intermitentes se fizeram contingentes \ principalmente na transição da fase em que a irmã bem tratava a cliente para a fase em que começaram as punições.

Os comportamentos de organização/limpeza foram diferencialmente reforçados, modelando os comportamentos de esquiva da cliente. "O" tentava desempenhar o trabalho melhor do que havia acabado de fazer a ponto de apresentar auto-regras relacionadas ao seu desempenho que especificavam um aumento na freqüência e intensidade dos comportamentos de esquiva.

Na época a classe de respostas relacionada à compulsão estava circunscrita aos comportamentos de organização/limpeza da casa e tinha como função se esquivar das punições da irmã. Após sair da casa da irmã e com o passar dos anos, ocorreu uma mudança no controle operante das respostas compulsivas. O controle exercido pelos eventos antecedentes, a exemplo da casa suja, e conseqüentes, a exemplo da esquiva das punições da irmã, foi transferido para outros estímulos. Isso ocorreu com o pareamento dos eventos antecedentes e conseqüentes com outros estímulos através da generalização de estímulos ou formação de classes de estímulos equivalentes (Zamignani, 2001 b). Na ocasião do início dos atendimentos, não somente os serviços domésticos estavam implicados, mas também respostas relacionadas à manufatura e venda dos bombons. Ao longo do tempo, os eventos antecedentes aos comportamentos compulsivos de manufatura/venda dos bombons também se tornaram variáveis controladoras. Em conjunto, as respostas de organização/limpeza da casa e de manufatura/venda dos bombons vieram a formar uma classe comportamental mais abrangente, que passou a ter como conseqüência a esquiva das atuais preocupações cotidianas referidas nas queixas. Também, na vigência de um ambiente com pouca estimulação aversiva, outras conseqüências passaram a exercer o controle operante da cadeia comportamental, a exemplo dos reforços sociais apresentados pelos familiares da cliente por ocasião do reconhecimento de um lar limpo e organizado.

\section{Considerações Finais}

Os eventos distais pelos quais a cliente passou vieram a tocar em uma interessante questão referente ao TOC: por que alguém que passou pelo condicionamento da ansiedade desenvolveria o TOC e não outro transtorno de ansiedade? A história de aprendizagem dos comportamentos relacionados ao TOC traz uma peculiaridade que o diferencia dos outros transtornos de ansiedade.

Em transtornos como a fobia específica ou o transtorno do pânico há a possibilidade de fuga/esquiva 
dos eventos aversivos (Kaplan, Sadock \& Greeb, 2003). Alguém que tenha fobia de elevadores conseguirá esquivar-se deles com sucesso. Esquivas efetivas também são observadas nas evitações agorafóbicas normalmente encontradas nos indivíduos com o transtorno do pânico (Craske \& Barlow, 1999). Semelhante fato não ocorreria na história de condicionamentos do TOC. A característica diferenciadora desse transtorno é o fato de o comportamento a ser punido ser o próprio comportamento de esquiva emitido. Em outras palavras, os comportamentos compulsivos no TOC freqüentemente não esquivam o estímulo aversivo contingente, mas o levam até ele. A recorrente emissão desse operante é justificável frente às contingências de reforçamento negativo vigentes, sejam elas naturais, como no caso das contingências às quais " $\mathrm{O}$ " esteve exposta, ou sejam elas acidentais, como ocorre nas associações causais verificadas no comportamento supersticioso (Skinner, 1953/1965).

Estendendo o raciocínio para os outros padrões sintomáticos do TOC, poderiam ainda ser abordados os dois tipos clássicos: os checadores e os lavadores de mãos (American Psychiatric Association, 2003). Os condiciona-mentos dos operantes compulsivos e dos respondentes ansiosos nesses tipos sintomáticos poderiam também ocorrer através de mais de um pareamento do aversivo primário com os aversivos secundários. Essa recorrência de eventos aversivos ocorreria normalmente durante a infância em práticas parentais que utilizam a coerção como meio de educar os filhos (Gomide, 2003). Do mesmo modo, hipotetizase que um evento aversivo único poderá dar origem ao transtorno. Kaplan et al. (2003) afirmaram que mais de 50\% dos pacientes experimentaram início súbito dos sintomas; aproximadamente $50 \%$ a $70 \%$ o tiveram após um acontecimento estressante, tal como gravidez, morte de um parente ou problema sexual. Se o evento eliciar intensa ansiedade no organismo, a possibilidade se fará provável. Durante o início da aprendizagem, um futuro checador poderia vir a associar o seu inadequado desempenho prévio do comportamento de checar à causa para uma punição vivenciada. Alguém que tivesse sua casa violada e roubada, estando sua família sob ameaça direta, poderia associar a checagem inadequada de todas as portas e janelas como sendo a causa do evento. O mesmo ocorreria com os lavadores quando do surgimento de uma doença séria na família. Após o evento aversivo, é provável que o seu comportamento de lavar torne-se mais intenso e freqüente em uma próxima emissão. Com a generalização, o controle operante dos comportamentos compulsivos se estenderá para outros eventos antecedentes e conseqüentes. Outrossim, os comportamentos de esquiva também deverão ser efetivos algumas vezes, posto que há a necessidade do reforço intermitente para que não entrem em extinção operante.

A despeito do caráter especulativo trazido na hipótese sobre os condicionamentos noTOC, seu estudo detalhado certamente merecerá experimentações controladas de mesma qualidade das questões que suscita. Em especial, percebeu-se a pertinência do estudo do controle exercido pelo comportamento verbal.

\section{Referências}

American Psychiatric Association. (2003). Manual diagnóstico e estatístico de transtornos mentais - DSM-IV-TR, Porto Alegre: Artmed.

Abreu, P.R., \& Prada, C.G. (2004). Transtorno de ansiedade obsessivo-compulsivo e transtorno da personalidade obsessivo-compulsivo: um "diagnóstico" analítico-comportamental. Revista Brasileira de Terapia Comportamental e Cognitiva, 6 (2), 211-220.

Busatto, G.F., Zamignani, D.R., Buchpiguel, C.A., Garrido, G.E., Glabus, M.F., \& Rocha, E.T. (2000). A voxel-based investigation of regional cerebral blood flow abnormalities in obsessive-compulsive disorder using single photon emission computed tomography (SPECT). Psychiatry Research: Neroimaging, 99 (1), 15-27.

Catania, A.C. (1999). Aprendizagem: comportamento, linguagem e cognição. Porto Alegre: Artmed.

Cavalcante, S.N., \& Tourinho, E.Z. (1998). Classificação e diagnóstico na clínica: possibilidades de um modelo analítico-comportamental. Psicologia:Teoria e Pesquisa, $14(1), 139-147$.

Craske, M.G., \& Barlow, D.H. (1999). Transtorno do pânico e agorafobia. In D.H. Barlow (Org.). Manual clínico dos transtornos psicológicos (pp. 13-62). Porto Alegre: Artmed.

Del-Porto, J.A. (2001). Epidemiologia e aspectos transculturais do transtorno obsessivo-compulsivo. Revista Brasileira de Psiquiatria, 23 (Supl 2), 3-5.

Del-Prette, A., \& Bolsoni-Silva, A.T. (2003). Problemas de comportamento: um panoroma da área. Revista Brasileira de Terapia Comportamental e Cognitiva, 5 (2), 91-103.

Estes, W.K., \& Skinner, B. F. (1941). Some quantitative properties of anxiety. Journal of Experimental Psychology, 29 (1), 390-340. 
Gomide, P. (2003). Estilos parentais e comportamento anti-social. In A. Del Prette \& Z. Del Prette (Orgs). Habilidades sociais, desenvolvimento e aprendizagem. Questões conceituais, avaliação e intervenção (pp. 21-60). São Paulo: Alínea.

Gongora, M.A.N. (2003). Noção de psicopatologia na análise do comportamento. In C.E. Costa, J.C. Luzia \& H.H.N. Sant'Anna (Orgs). Primeiros passos em análise do comportamento e cognição (pp. 93-109). Santo André: ESETec.

Gonzalez, C.H. (1999). Transtorno obsessivo-compulsivo. Revista Brasileira de Psiquiatria, 21 (Supl 2), 29-32.

Gonzalez, C.H. (2001). Aspectos genéticos do transtorno obsessivo-compulsivo. Revista Brasileira de Psiquiatria, 23 (Supl 2), 38-41.

Hounie, A.G., Brotto, S.A., Diniz, J., Chacon, P.J., \& Miguel, E.C. (2001). Transtorno obsessivo-compulsivo: possíveis subtipos. Revista Brasileira de Psiquiatria, 23 (Supl 2), 13-16.

Jenike, M.A. (2004). Obsessive-compulsive disorder. The New England Journal of Medicine, 350 (3), 259-265.

Kaplan, H.I., Sadock, B.J., \& Greeb, J.A. (2003). Compêndio de Psiquiatria. Ciências do comportamento e psiquiatria clínica (7.ed.). Porto Alegre: Artmed.

Lacerda, A.L.T. (2000). Transtorno obsessivo-compulsivo: um estudo psicopatológico, neuropsicológico e de fluxo sangüíneo cerebral regional (HMPAO-Tc99m). Tese de doutorado não-publicada, Universidade Estadual de Campinas.

Lacerda, A.L.T., Dalgalarrondo, P., \& Camargo, E.E. (2001). Achados de neuroimagem no transtorno obsessivo-compulsivo. Revista Brasileira de Psiquiatria, 23 (Supl 1), 24-27.

Lundin, R.W. (1977). Personalidade: uma análise do comportamento. São Paulo: Heider. (Originalmente publicado em 1969)

Marazziti, D., \& Di Nasso, E. (2000). What pharmacology teaches us about the pathophysiology of obsessivecompulsive disorder. Revista Brasileira de Psiquiatria, 22 (4), 185-188

Marques, C. (2001). Tratamento farmacológico do transtorno obsessivo-compulsivo. Revista Brasileira de Psiquiatria, 23 (Supl 2), 49-51.

Marinho, M.L. (2001). O modelo médico e o modelo psicológico. In R.C. Wielenska (Org.). Sobre comportamento e cognição. Questionando e ampliando a teoria e as intervenções clínicas e em outros contextos (pp.143-145). Santo André: ESETec.

Miguel, E.C., Rauch, S.L., \& Jenike, M.A. (1998). Transtorno obsessivo-compulsivo. In J.F. Leckman (Org.). Neuropsiquiatria dos gânglios da base (pp. 237-262). São Paulo: Lemos Editorial \& Gráficos.

Parmet, S.; Glass, T.J., \& Glass, R. (2004). Obsessive-compulsive disorder. The Journal of the American Medical Association, 292 (16), 2040.

Pujol, J., Soriano-Mas, C., Alonso, P., Cardorner, N., Menchón, J., Deus, J., \& Vallejo, J. (2004). Mapping structural brain alterations in obsessive-compulsive disorder. Archives of General Psyquiatry, 61 (7), 720-730.

Reese, E.P. (19681978) Análise do comportamento humano. Rio de Janeiro: Livraria José Olympio.

Ronchetti, R., Böhme, E.S., \& Ferrão, Y.A. (2004). A hipótese imunológica no transtorno obsessivo-compulsivo: revisão de um subtipo (PANDAS) com manifestação na infância. Revista de Psiquiatria do Rio Grande do Sul, 26 (1), 62-69.

Sério, T.M.A.P., Andery, M.A., Gioia, P.S., \& Micheletto, N. (2004). Os conceitos de discriminação e generalização. In T.M.A.P. Sério, M.A. Andery, P.S. Gioia \& N. Micheletto (Orgs.). Controle de estímulos e comportamento operante. Uma (nova) Introdução (pp. 7-24). São Paulo: EDUC.

Skinner, B.F. (1965). Science and human behavior. New York: Free Press. (Originalmente publicado em 1953)

Skinner, B.F. (1976). About behaviorism. New York: Appleton-Century. (Originalmente publicado em 1974)

Skinner, B.F. (1984). Selection by consequences. The Behavioral and Brain Sciences, 7 (1), 477-510. (Originalmente publicado em 1981)

Skinner, B.F. (1990). Can Psychology be a science of mind? American Psychologist, 45 (11), 1206-1210.

Skinner, B.F. (2002). Questões recentes na análise do comportamento. Campinas: Papirus. (Originalmente publicado em 1989)

Torres, A.R., \& Prince, M.J. (2004). A importância de estudos epidemiológicos sobre o transtorno obsessivo-compulsivo. Revista Brasileira de Psiquiatria, 26 (3), 141-142.

Tourinho, E.Z. (1999). Conseqüências do externalismo behaviorista radical. Psicologia: Teoria e Pesquisa, 15 (2), 107-115.

Tourinho, E.Z., Teixeira, E.R., \& Maciel, J.M. (2000). Fronteiras entre análise do comportamento e fisiologia: Skinner e a temática dos eventos privados. Psicologia: Reflexão e Crítica, 13 (3), 425-434.

Valente Jr, A.A., \& Filho, G.B. (2001). Aspectos neurorradiológicos do transtorno obsessivo-compulsivo: - papel dos gânglios da base. Revista Brasileira de Psiquiatria, 23 (Supl 2), 42-45.

Zamignani, D.R. (2001a). Uma tentativa de caracterização da prática clínica do analista do comportamento no atendimento de clientes com e sem o diagnóstico de transtorno obsessivo-compulsivo. Dissertação de mestrado não-publicada, Pontifícia Universidade Católica de São Paulo.

Zamignani, D.R. (2001b). Uma tentativa de entendimento do comportamento obsessivo-compulsivo: algumas variáveis negligenciadas. In R.C. Wielenska (Org.). Sobre comportamento e cognição. Questionando e ampliando a teoria e as intervenções clínicas em outros contextos (pp. 247-256). Santo André: ESETec.

Recebido para publicação em 5 de novembro de 2004 e aceito em 2 de junho de 2005. 